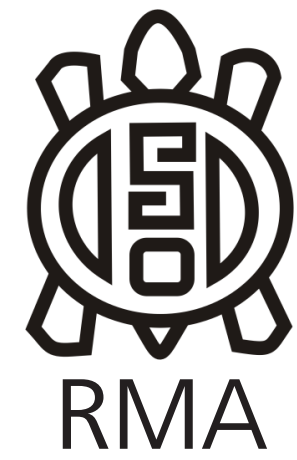

Arqueología

\title{
Uso del espacio y organización tecnológica en la Colonia El Chalía, sudoeste de Chubut, Argentina
}

\author{
Land use and technological organization in Colonia El Chalía, \\ southwest Chubut, Argentina
}

\author{
Analía Castro Esnal*, Lucía A. Gutiérrez ${ }^{* *}$, Florencia E. Ronco*** y
} Cecilia Pérez de Micou****

*Consejo Nacional de Investigaciones Científicas y Técnicas (CONICET).Facultad de Filosofía y Letras, Universidad de Buenos Aires (FFyL, UBA). Instituto Nacional de Antropología y Pensamiento Latinoamericano (INAPL). 3 de Febrero 1378 (1426), Ciudad Autónoma de Buenos Aires, Argentina.

E-mail: analiacastro@gmail.com

**Facultad de Filosofía y Letras, Universidad de Buenos Aires (FFyL, UBA). Instituto Nacional de Antropología y Pensamiento Latinoamericano (INAPL). 3 de Febrero 1378 (1426), Ciudad Autónoma de Buenos Aires, Argentina. E-mail: luciagutierrez94@gmail.com ***Facultad de Filosofía y Letras, Universidad de Buenos Aires (FFyL, UBA). Instituto Nacional de Antropología y Pensamiento Latinoamericano (INAPL). 3 de Febrero 1378 (1426), Ciudad Autónoma de Buenos Aires, Argentina. E-mail: florenciae.ronco@gmail.com

${ }^{* * * *}$ Consejo Nacional de Investigaciones Científicas y Técnicas (CONICET).Facultad de Filosofía y Letras, Universidad de Buenos Aires (FFyL, UBA). Instituto Nacional de Antropología y Pensamiento Latinoamericano (INAPL). 3 de Febrero 1378 (1426), Ciudad Autónoma de Buenos Aires, Argentina. E-mail: c.perezdemicou@yahoo.com.ar

\begin{abstract}
Resumen
Las investigaciones arqueológicas realizadas en el sudoeste de Chubut señalan que habría sido ocupado de modo continuo, aunque con diferencias locales, desde el Holoceno temprano hasta el presente. Ubicada sobre un corredor de movilidad mencionado en fuentes etnohistóricas, la Colonia El Chalía es una reserva indígena habitada en la actualidad por descendientes directos de grupos cazadores recolectores del siglo XIX. Los estudios arqueológicos efectuados indican su ocupación al menos desde el Holoceno tardío y caracterizan al área como un conjunto de paraderos de reutilización frecuente, de permanencia prolongada y con alta diversidad de actividades representadas. Con el objetivo de ampliar la información producida hasta el momento para la comprensión de los modos de habitar este espacio en el pasado, en este trabajo se presentan los resultados obtenidos a partir del análisis del material lítico de superficie proveniente de nuevas prospecciones. Se observa una variabilidad destacada entre sitios cercanos, por lo que se discute y complejiza esta caracterización originalmente formulada y se evalúan concordancias y/o discordancias con lo postulado en trabajos anteriores acerca de las estrategias tecnológicas aplicadas en lo referente al uso de materias primas y distribución en el espacio de actividades de manufactura de instrumentos líticos.
\end{abstract}

Palabras clave: Patagonia central; Reserva indígena; Movilidad; Uso del espacio; Tecnología lítica.

\begin{abstract}
Archaeological research in the southwest of Chubut indicates that it would have been occupied continuously, although with local differences, from the early Holocene to the present. Located on a mobility corridor mentioned in ethnohistoric sources, Colonia El Chalía is an indigenous reserve currently inhabited by direct descendants of nineteenth-century hunter-gatherers groups. Archaeological studies carried out indicate its occupation at least since the late Holocene and characterize the area as a set of settlements with frequent reuse, prolonged permanence and a high diversity of activities represented. To expand the information produced so far for the understanding of the ways of inhabiting this space in the past, this paper presents the results obtained from the analysis of ithic surface material from new surveys. An outstanding variability is observed between nearby sites, therefore the originally formulated characterization is discussed and complexed, and concordance and/or disagreement with the postulated in previous works about the technological strategies applied concerning the use of raw materials and distribution in the space of manufacturing activities of lithic instruments are evaluated.
\end{abstract}

Keywords: Central Patagonia; Indigenous reserve; Mobility; Land use; Lithic technology. 
La Colonia El Chalía es un área reservada concedida en 1916 por el Estado nacional a la comunidad del cacique Manuel Quilchamal, que ya la habitaba tradicionalmente y que lo sigue haciendo hasta la actualidad. El área formalmente concedida fue de 60.000 hectáreas, sin embargo posteriormente fue reducida a 32.902 hectáreas (Muñiz y Perea, 2000; Pinotti, 2001; Pinotti, 2004). La información histórica proveniente de viajeros, que mencionan que esta zona era atravesada por una ruta indígena e incluso dicen haber conocido allí a Quilchamal y su gente a fines del siglo XIX (Castro Esnal, 2010; 2014), llevó a plantear estudios arqueológicos en el área en el marco de proyectos de investigación centrados en el estudio de la movilidad y el uso del espacio por parte de los cazadores recolectores de la Patagonia central. Los trabajos arqueológicos desarrollados allí desde el año 2007 han dado cuenta de sitios que presentan de manera conjunta materiales de manufactura indígena y criollo/ europea, con una predominancia de desechos de talla lítica. Al tratarse en su totalidad de material arqueológico en superficie se les ha asignado una cronología tardía a partir de diversos indicadores: el material cerámico indígena (tres tiestos fechados: $461 \pm 35$ AP; $374 \pm 38$ AP y 471 38 AP; Castro Esnal, 2010); la tipología del material lítico recuperado; la presencia de materiales indígenas formatizados sobre vidrio y de artefactos de factura criollo/europea; y las fuentes escritas (Pérez de Micou, Sacchi, Castro y Funes, 2009; Castro Esnal, 2010; Castro Esnal, Sacchi y Pérez de Micou, 2011a; Sacchi, 2012; Castro Esnal, 2014; Casanueva, Castro Esnal y Pérez de Micou, 2019). No obstante, posteriores investigaciones arqueológicas de sitios estratificados en áreas cercanas, como Casa de Piedra de Roselló en Aldea Beleiro (Castro Esnal, Pérez de Micou y Casanueva, 2017a) y Alero Dasovich en Río Mayo (Aguerre, Andrieu y lantanos, 2017), advierten sobre una ocupación del sudoeste de Chubut desde el Holoceno temprano.

A partir de los sitios estudiados inicialmente en la colonia (sitios Laguna Quilchamal y Tapera Almeida; Figura 1) se establecieron tendencias tecnológicas con respecto a las estrategias utilizadas, especialmente en lo referente al uso de materias primas y a la distribución en el espacio de las actividades de manufactura, uso, descarte y reparación de instrumentos (Castro Esnal, 2010, 2014). De este modo, se caracterizó al área como un conjunto de paraderos de reutilización frecuente y de permanencia prolongada, en los que se encuentran representadas todas las etapas de manufactura de instrumentos, pero en especial las últimas etapas de confección y mantenimiento. Al mismo tiempo, se observó una alta diversidad de artefactos que indicaría una también alta diversidad de actividades llevadas a cabo en los sitios (Castro Esnal, 2010, 2014). Además, se realizaron análisis geoquímicos para establecer la fuente de procedencia de los artefactos confeccionados sobre obsidiana (materia prima alóctona) cuyos resultados señalaron el uso predominante de obsidiana de Pampa del Asador (Stern, 1999, 2004; Espinosa y Goñi, 1999), en el noroeste de la provincia de Santa Cruz (Castro Esnal, Pérez de Micou y Stern, 2011b; Stern, Castro Esnal, Pérez de Micou, Méndez y Mena, 2013).

En este trabajo se presentan nuevos resultados de análisis tecno-morfológicos realizados en muestras de material lítico de superficie provenientes de otros dos sitios prospectados en la colonia (Tapera Pescán y Casa Tolkin) con el fin de integrar esta información a la ya producida hasta el momento y discutir concordancias y/o discordancias con lo postulado en trabajos anteriores acerca de la caracterización de los sitios de esta zona y su articulación con los circuitos de movilidad macroregionales (Castro Esnal, 2010, 2014).

\section{Antecedentes arqueológicos del sudoeste de Chubut y áreas vecinas}

Los antecedentes arqueológicos conocidos del SO de Chubut y áreas vecinas, muestran la ocupación de ciertos espacios desde el Holoceno temprano, como el caso de Casa de Piedra de Roselló en Aldea Beleiro (Castro Esnal et al., 2017a) y Alero Dasovich en Río Mayo (Aguerre et al., 2017), en el So de Chubut, y de Baño Nuevo 1 (Mena, Lucero, Reyes, Trejo y Velásquez, 2000; Mena, Reyes, Stafford y Southon, 2003), El Chueco 1 (Reyes, Méndez, Trejo y Velásquez, 2007; Méndez et al., 2011) y Cueva de La vieja (Méndez et al., 2018), en Aysén (Chile). Otros sectores evidencian ocupación recién a partir de los 5000 años $\mathrm{AP}$, como el área de Bajo de Sarmiento (Moreno, Pérez y Ramírez, 2016), o más tardíamente, como el caso de Alero de las Manos Pintadas ubicado cerca del paraje Las Pulgas (Aschero, 1975a; Gradin y Aschero, 1978).

Otras áreas aledañas tales como el NO de Santa Cruz hacia el sur (Mengoni Goñalons, Figuerero Torres y Fernández, 2009; Mengoni Goñalons, Fernández y Figuerero Torres, 2013) o los valles del Pico y Apeleg hacia el norte (Scheinsohn, Rizzo y Leonardt, 2010; Rizzo, Scheinsohn y Leonardt, 2016) presentan ocupaciones con fechados del Holoceno tardío; aunque el reciente fechado de restos humanos procedentes del valle del Genoa advierte la presencia humana allí desde el Holoceno medio (Scheinsohn et al., 2017).

Esta información junto con la presencia en la Colonia El Chalía de descendientes directos de los grupos cazadores recolectores del siglo XIX sugiere que esta área de la Patagonia central habría sido ocupada de modo continuo, aunque con diferencias locales, desde el Holoceno temprano hasta el presente.

\section{El área de investigación}

La Colonia El Chalía se encuentra habitada en la actualidad por los descendientes de la comunidad del cacique Manuel Quilchamal. Este grupo indígena vio reducido su territorio tras la denominada "Conquista del Desierto", 
cuya presión política y militar los habría circunscripto a esta zona, restringiendo de esta manera su movilidad residencial. La ocupación por parte de la población tradicional ha sido continua desde entonces, aunque el número inicial de hectáreas adjudicadas en 1916 se ha reducido debido a los avances de estancieros de campos aledaños. La colonia está ubicada en el departamento de Río Senguer, al sudoeste de Chubut (Figura 1) y es la única de la zona cuyos habitantes se adscriben como "tehuelches".

\section{Características ambientales}

Es una zona esteparia localizada en el área de contacto entre la Patagonia semiárida (al Este) y la provincia Subantártica (al Oeste). Desde el punto de vista fitogeográfico se trata de una estepa arbustiva graminosa que pertenece al Distrito Occidental (Soriano, 1956), donde predominan los vientos del oeste, con una temperatura media anual entre 7 y $9{ }^{\circ} \mathrm{C}$ y lluvias que alcanzan los $200 \mathrm{~mm}$ anuales. La Pampa del Chalía se extiende sin interrupción hacia el este perdiendo gradualmente altura y es cruzada, de oeste a este, por el río Mayo. A él se une el arroyo Chalía que reúne una cantidad de arroyuelos. La laguna Quilchamal pertenece a la misma cuenca del lago Blanco, ambos constituyen los restos de un lago glacial de mucha mayor extensión (González, 1978).

Desde el punto de vista geológico, esta región se caracteriza principalmente por el gran desarrollo de las unidades sedimentarias terciarias aflorantes en los faldeos de las mesetas y en las márgenes de los principales ríos. Hacia el oeste, en la zona limítrofe con Chile, se encuentran las unidades volcánicas jurásicas y cretácicas que constituyen cerros aislados, relictos de la erosión glacial, y hacia el norte, los afloramientos sedimentarios cretácicos que forman cerros y mesetas y que en algunos casos rematan en coladas basálticas terciarias (Dal Molin, 1998).

Las materias primas líticas adecuadas para la talla disponibles localmente son rodados acarreados por procesos glacio-fluviales, provenientes de rocas más antiguas ubicadas en el oeste de la región, y otras rocas sedimentarias. Los afloramientos de rocas efusivas básicas (del Eoceno) son escasos en la región y muy distantes entre sí. El afloramiento de este tipo que se encuentra más cercano a El Chalía (20 km) es el de Loma Redonda (González, 1978).

\section{El Chalía en las fuentes históricas del siglo XIX y posteriores abordajes científicos}

La redundancia en la mención de esta área por parte de viajeros de fines del siglo XIX y principios del siglo $X X$ da cuenta de su relevancia para esos momentos (Castro Esnal, 2010; 2014). Acompañado por un grupo de tehuelches, George Musters (1871/1964) fue el primer europeo en recorrer la zona, y menciona haber acampado en el paradero Tele, dentro de la actual área de la colonia. Posteriormente, el explorador galés Llwyd ap Iwan visitó El Chalía en tres oportunidades, entre los años 1893 y 1897 (Roberts y Gavirati, 2008); es el primero en realizar descripciones detalladas de su paso por la zona y en usar el topónimo Chalía, aparentemente referido al chaquil (Alstroemeria sp.) o a la challa (Tropeolum patagonicum) (Pérez de Micou, 1994), dos especies muy apreciadas por sus tubérculos comestibles, disponibles a fines de la primavera. En 1896, Henry de la Vaulx encuentra una explicación similar con respecto a este topónimo, en su caso para el Arroyo Chalía (de la Vaulx, 2008).

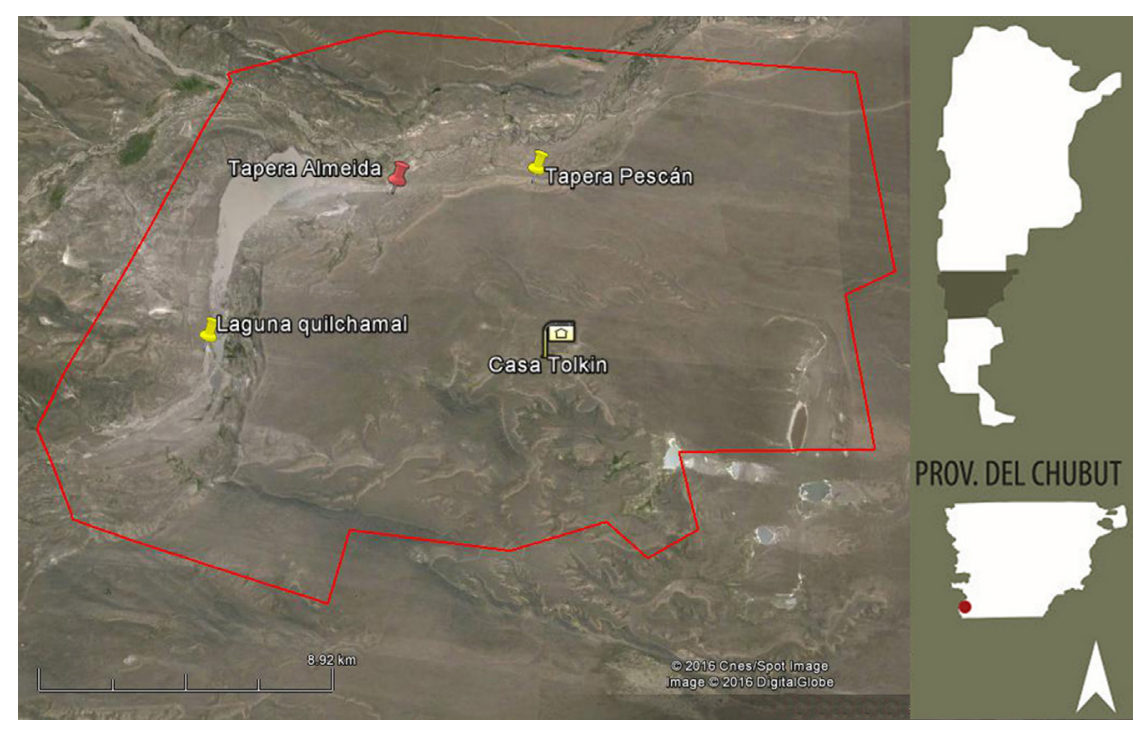

Figura 1. Colonia El Chalía, sudoeste de Chubut, y sitios arqueológicos trabajados.

Figure 1. Colonia El Chalia, SW Chubut, and location of archaeological sites mentioned. 
Las descripciones sobre la vida cotidiana de la comunidad continúan en 1904 con Onelli, quien vivió varios días en la toldería del cacique Quilchamal, ubicada en aquel momento en la zona del Guenguel (Onelli, 1998). Además, según Escalada (1949) los viajeros Moreno, Moyano, Lista, Mayo, Kozlowsky, Ameghino y Burmeister habrían estado en contacto con Quilchamal luego de la "Conquista del Desierto", aunque este autor no menciona expresamente cuáles son los documentos en los que dichos exploradores refieren al cacique. Por su parte, Fontana, nombrado gobernador del territorio del Chubut en 1884, publicó descripciones hidrogeográficas del área, relevadas en su expedición del año 1886 (Fontana, 1886).

En la actualidad, las investigaciones científicas en la Colonia El Chalía han sido abordadas inicialmente desde la Antropología Social, y dan cuenta de factores lingüísticos, económicos y simbólicos, entre otros (Fernández Garay y Hernández, 1999; Muñiz y Perea, 2000; Pinotti, 2001, 2004; Miceli y Guerrero, 2007; García, 2009). La primera aproximación arqueológica fue realizada por Pérez de Micou y equipo en el año 2007 (Pérez de Micou et al., 2009).

\section{Metodología y sitios trabajados}

La elección de las zonas que se prospectaron en la colonia respondió principalmente a su accesibilidad, dada a partir del contacto con familias que viven actualmente allí, quienes dieron su permiso para trabajar en los alrededores de sus viviendas y en algunos casos incluso acompañaron las tareas de campo. Previamente, se habían mantenido reuniones con el cacique de la comunidad y con su representante comunitario. La metodología utilizada consistió en la prospección de las áreas próximas a fuentes de agua: el arroyo Chalía, la laguna Quilchamal y manantiales cercanos a viviendas actuales y a taperas.

En un primer momento se registraron sitios ubicados a orillas de la laguna Quilchamal y en la tapera de Almeida, situada en las cercanías de un manantial (Castro Esnal, 2010). A partir de posteriores trabajos de campo se localizaron dos nuevos sitios cuyos resultados se presentan en este trabajo: Tapera Pescán y Casa Tolkin (Figura 1).

Tapera Pescán consiste en una concentración de material lítico superficial, en una gran hoyada de deflación cercana a los restos de un asentamiento del siglo XX (Figura 2a y c). Aunque los restos de la casa aparentemente no se han conservado, se observaron una alameda y escasos restos de materiales criollo/europeos en superficie (un artefacto de cuero; una herradura; un trozo de metal; cinco fragmentos de vidrio) (Casanueva et al., 2019). Tanto el área en la que habría estado la tapera como el manantial se ubican en una cota inmediatamente superior a la hoyada de deflación en donde se hallaron los materiales arqueológicos, por lo que no puede descartarse una migración de materiales desde áreas más elevadas. El arroyo Chalía se encuentra a una distancia aproximada de $1 \mathrm{~km}$, en una cota inferior. Se realizó una recolección sistemática del material hallado en superficie mediante transectas recorridas por tres investigadores. La superficie recorrida fue de aproximadamente $50.000 \mathrm{~m}^{2}$ y se recuperó la totalidad del material hallado.

En Casa Tolkin se relevó la presencia de material arqueológico en los alrededores de una casa actualmente habitada (Figura 2d). Se trata en su mayoría de material lítico y en menor número fragmentos de gres $(n=3)$ y vidrio (13 fragmentos, tres de ellos con posibles retoques, y 3 lascas) (Casanueva et al., 2019). En este caso el muestreo se efectuó en circunstancias relacionadas con la realización de entrevistas a los habitantes de la casa y no con un trabajo arqueológico planificado previamente. Las recolecciones fueron realizadas a instancias de los pobladores, que se dispusieron a colaborar con las tareas de campo, al mismo tiempo que hacían comentarios sobre algunas características de los materiales hallados. Dadas estas condiciones, la muestra será trabajada tomando las precauciones metodológicas del caso y teniendo en cuenta que no se recogió el total de artefactos observados por nosotros. Es necesario tener presente, además, que en este y otros sitios, los pobladores han formado pequeñas colecciones de material lítico, al cumplir con sus tareas habituales en el campo. De todos modos, se consideró que el análisis de la muestra recuperada en Casa Tolkin aportará información relevante para los trabajos de investigación arqueológica en la colonia. La superficie prospectada fue de aproximadamente $60.000 \mathrm{~m}^{2}$.

El análisis de laboratorio se realizó siguiendo los lineamientos de Aschero (1975b; 1983). Debido a las características de la recolección en Casa Tolkin se consideraron sólo las variables generales al momento de efectuar comparaciones con los conjuntos obtenidos en otros sitios de El Chalía. Las materias primas fueron caracterizadas macroscópicamente según categorías amplias.

\section{Resultados}

En los conjuntos recuperados en Tapera Pescán (TP) y Casa Tolkin (CT) predominan los desechos de talla (Tabla 1), siendo 373 (75\%) de un total de 500 artefactos líticos recolectados. La densidad de hallazgos (cantidad de artefactos por $\left.\mathrm{m}^{2}\right)$ es mayor en $\mathrm{CT}(0,0032)$ que en TP $(0,0028)$. Esta diferencia se acentúa si se toma en cuenta que en CT no se recolectó la totalidad del material, por lo que la densidad real se estima que es aún mayor. El estado general de fragmentación de la muestra es prácticamente similar en ambos sitios: el $71 \%$ de los artefactos de TP se encuentran fracturados y el $69 \%$ en CT.

Con respecto a los desechos de talla, en ambos conjuntos predominan las lascas angulares (en TP representan el 

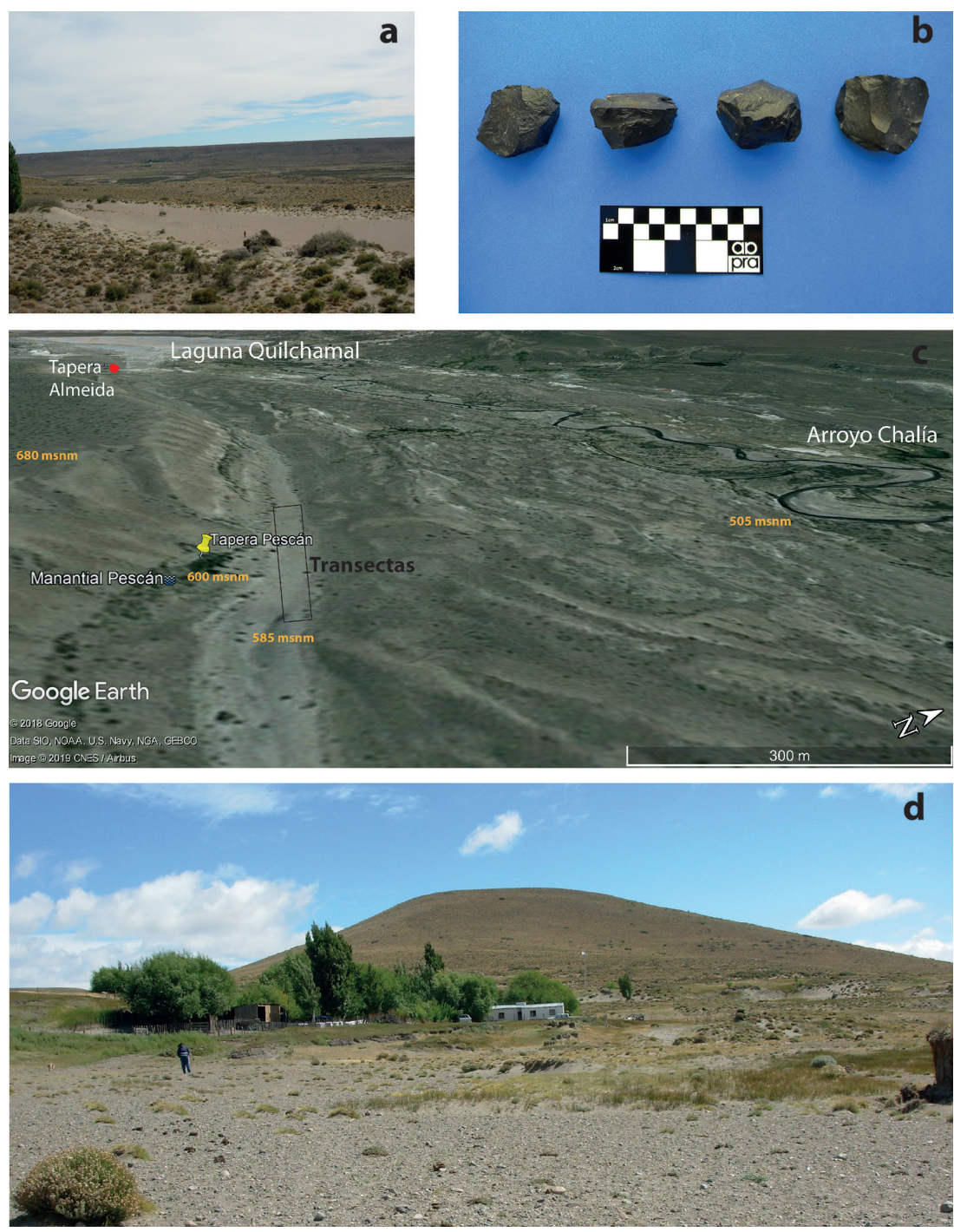

Figura 2. a: Tapera Pescán; b: muestra de Núcleos de Tapera Pescán; c: vista en perspectiva del sitio Tapera Pescán; d: Casa Tolkin.

Figura 2. a: Tapera Pescán; b: core sample from Tapera Pescán; c: Tapera Pescán site in perspective; d: Casa Tolkin.

$59 \%$ y en CT el 68\%, de las lascas enteras). Las lascas de formatización final y de reactivación de filos de instrumentos están prácticamente ausentes (2 lascas de adelgazamiento/reducción bifacial en TP y 1 de reactivación de filo en $\mathrm{CT}$ ). En TP se destaca la vulcanita básica como materia prima y en CT esta materia prima se presenta en una frecuencia levemente menor que las rocas silíceas (Tabla 2). En cuanto a las categorías de tamaño (sensu Bagolini modificado en Aschero, 1975b, 1983) se observa que las lascas de tamaños muy pequeños son escasas y hay una mayor variabilidad para los desechos de vulcanita básica en ambos conjuntos (Figura 3a). Los talones más representados en ambas muestras son los lisos, aunque en TP es importante la cantidad de talones facetados para el caso de las vulcanitas básicas (Figura 3b). Las mayores proporciones de lascas con reserva de corteza se dan también en la vulcanita básica (Figura 3c).
Entre los artefactos formatizados de ambos sitios (en total 86 instrumentos formatizados por percusión y 5 con técnicas de picado/abrasión y pulido) predominan los instrumentos unifaciales (Tabla 1) sobre rocas silíceas (Tabla 2). En TP es interesante la representación de instrumentos formatizados sobre hoja (9 de 25); en cambio en CT predominan ampliamente las lascas angulares como forma base de los instrumentos (28 de 61), dando cuenta de técnicas de aprovechamiento diferencial de las materias primas en cada sitio. Se destaca la cantidad de raspadores en CT (en su mayoría de filo frontal corto). Las puntas de proyectil recuperadas son en su mayoría de limbo triangular: la punta de TP presenta fracturados su base y su limbo y las tres puntas de CT son pedunculadas (una de ellas es de obsidiana y presenta una alta reactivación en su limbo). 
Tabla 1. Artefactos líticos recuperados en Tapera Pescán (TP) y Casa Tolkin (CT).

Table 1. Lithic assemblage recovered from Tapera Pescán (TP) and Casa Tolkin (CT).

\begin{tabular}{|l|r|r|r|}
\hline \multicolumn{1}{|c|}{ Artefacto } & \multicolumn{1}{c|}{ TP } & \multicolumn{1}{c|}{ CT } & \multicolumn{1}{c|}{ Total } \\
\hline Artefacto de formatización sumaria & 2 & 4 & 6 \\
\hline Cuchillo & 7 & 9 & 16 \\
\hline Desecho de talla & 189 & 184 & 373 \\
\hline Filo Natural con Rastros Complement. & 5 & 4 & 9 \\
\hline Fragmento de instrumento bifacial n/d & - & 3 & 3 \\
\hline Fragmento de intrumento n/d & 2 & 6 & 8 \\
\hline Cuña & 1 & 1 & 2 \\
\hline Mano & - & 2 & 2 \\
\hline Muesca & 1 & 1 & 2 \\
\hline Núcleos & 13 & 14 & 27 \\
\hline Percutor & - & 1 & 1 \\
\hline Preforma de Bola & 1 & 1 & 2 \\
\hline Punta de proyectil & 1 & 3 & 4 \\
\hline Raedera & 4 & 7 & 11 \\
\hline Raspador & 6 & 27 & 33 \\
\hline RBO & 1 & - & 1 \\
\hline Total & 233 & 267 & 500 \\
\hline
\end{tabular}

Los núcleos se presentan en su mayoría enteros (sólo 5 son fragmentos) y son predominantemente de vulcanita básica) (Tabla 2) y amorfos (6 amorfos, 2 bipolares, 1 discoidal parcial, 1 poliédrico, 1 prismático parcial bidireccional, 1 prismático parcial unidireccional y 1 fragmento de núcleo en TP; y 8 amorfos, 3 bipolares, 1 lasca nodular, 1 prismático parcial bidireccional y 1 fragmento de núcleo en $\mathrm{CT}$ ). La totalidad de los núcleos de vulcanita básica de Tapera Pescán presentan reserva de corteza. Todos pesan menos que $100 \mathrm{~g}$ y en cuanto a su tamaño relativo son en su mayoría grandes (sensu Bagolini modificado en Aschero, 1975b; 1983) (Figura 2b).

\section{Discusión}

Los resultados aquí presentados muestran diferencias entre ambos sitios y con respecto a los sitios trabajados anteriormente en la Colonia El Chalía. Las investigaciones previas señalaban una representación importante de las lascas de las últimas etapas del proceso de manufactura, especialmente para Laguna Quilchamal; de acuerdo con esto fueron inferidas actividades de formatización final, reparación y/o reactivación de instrumentos (Castro Esnal, 2010; 2014). No obstante, en las nuevas muestras analizadas en el presente trabajo este tipo de desechos está escasamente representado. Por otro lado, se observa que Tapera Pescán presenta una densidad baja de material en comparación con el resto de los sitios y, sobre todo, con Laguna Quilchamal, en donde se encontró material concentrado en conjuntos con alta densidad -como por ejemplo la concentración Quil5 (Castro Esnal, 2010; 2014)
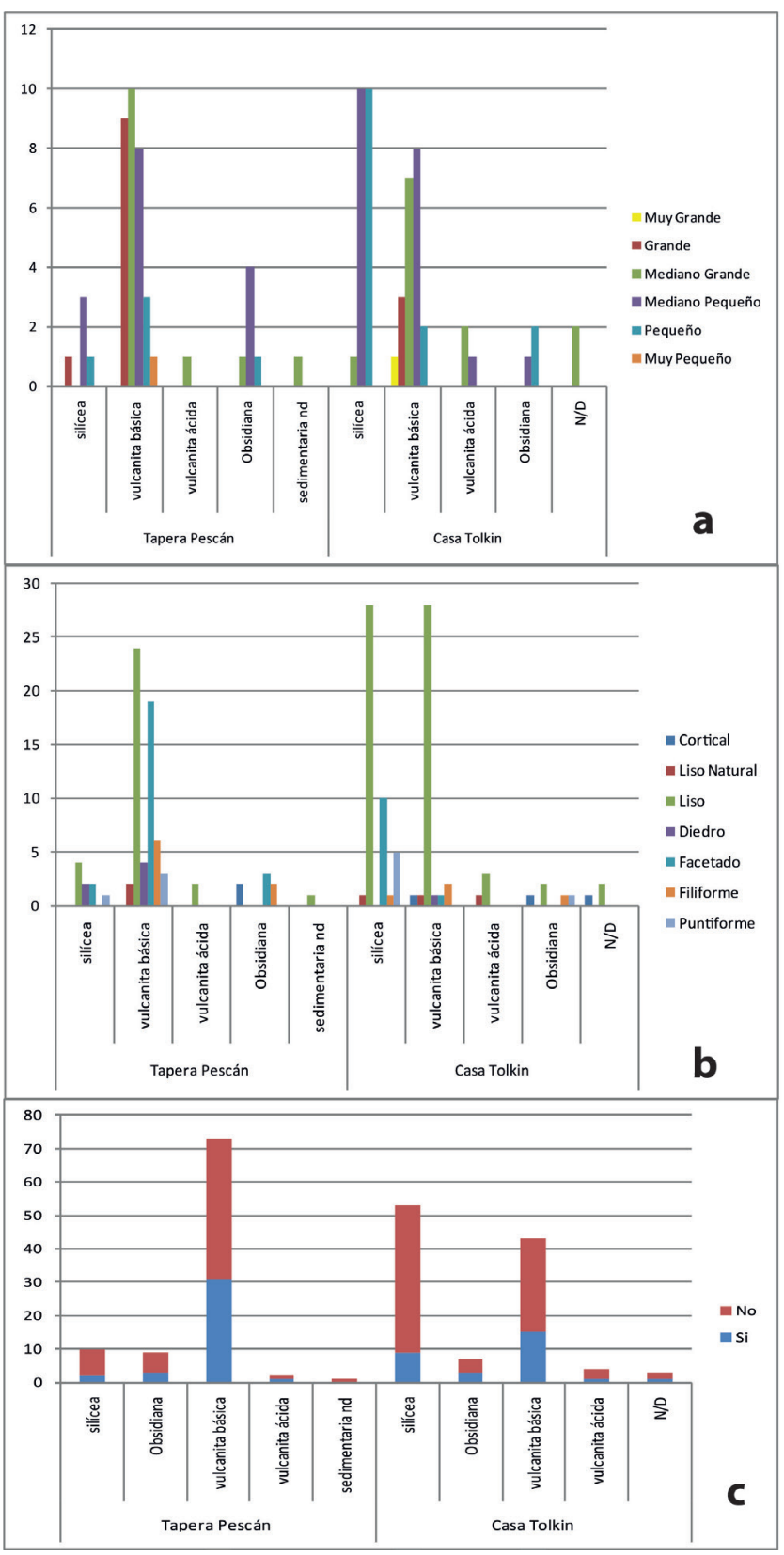

Figura 3. a: Tamaños desechos enteros por materia prima; b: Talones por materia prima de los desechos enteros; c: Presencia de corteza en los desechos de talla (enteros y fracturados con talón). El eje de ordenadas indica frecuencias absolutas.

Figura 3. a: Debitage size per raw material; $b$ : striking platforms per raw material of complete debitage; c: presence of cortex in debitage (complete and fragmented with striking platform). $Y$ axis indicates absolute frequencies.

que presenta una densidad de 29,25 artefactos por $\mathrm{m}^{2}$ (especialmente lascas pequeñas y microlascas)- y que es hasta el momento el único sitio en el que se hallaron restos de cerámica indígena. Cabe recordar que este sitio fue asentamiento de tolderías sin que se conozcan construcciones de adobe ni de otros materiales en este emplazamiento, estructuras que sí aparecen en otros sitios de la colonia y demuestran una sucesión de técnicas 
constructivas habitacionales durante el siglo XX (García y Moreno, 2010). Esto podría señalar una utilización diferencial de ciertos espacios de la colonia, aunque los procesos de formación de los distintos sitios trabajados podrían estar obliterando en algunos casos la presencia de lascas pequeñas.

Como se mencionó, todos los sitios estudiados tienen en común el estar estratégicamente ubicados junto a una fuente de agua, ya sea la laguna Quilchamal o manantiales, abundantes en la colonia. Sin embargo, en cuanto a su disposición en el paisaje, Casa Tolkin se diferencia de los demás por estar ubicado en una cota superior sobre la meseta del Chalía (664 m s.n.m). Preliminarmente, esta particularidad puede verse reflejada en las diferencias que presenta el conjunto lítico de este sitio con respecto a la representación de materias primas, especialmente en la mayor frecuencia de artefactos de sílice en comparación con lo observado para Tapera Pescán. Además, en Casa Tolkin, se destaca la abundancia de raspadores. Onelli (1998:75), en su capítulo sobre las tolderías de Quilchamal, indica cómo las mujeres recogían los raspadores de piedra para adelgazar pieles dado que no dominaban, como antiguamente, el arte de su confección, dando cuenta así de una reutilización de estas herramientas y la continuidad del trabajo sobre cuero. Hasta el momento de la recolección, la familia de Casa Tolkin continuaba realizando, no ya quillangos sino botas de potro y soguería en cuero. Estos datos podrían señalar no sólo una utilización diferencial de los sitios estudiados, sino además una continuidad en algunas labores a pesar de los cambios acaecidos en el siglo XX.

Además, en lo que refiere a las materias primas se destaca la frecuencia de vulcanitas básicas en Tapera Pescán, donde se observa un alto porcentaje de desechos de basalto de grano muy fino, de excelente calidad para la talla, y en una amplia variedad de tamaños. Este dato contrasta con los obtenidos anteriormente a partir de los otros sitios en la colonia en donde, en general, predominan los artefactos sobre sílices y, por el contrario, los de vulcanita básica se presentan en menor proporción (Castro Esnal, 2010; 2014). En este sitio, además, se destacan en esta materia prima la frecuencia de hojas como forma base, la presencia de núcleos discoidales y prismáticos parciales, y la frecuencia de talones facetados; estas variables dan cuenta en este conjunto de un aprovechamiento diferencial de las vulcanitas básicas. Cabe mencionar que en Tapera Pescán se registró un sector que concentra, de manera aparentemente antrópica, una importante cantidad de nódulos de vulcanitas básicas (aproximadamente 200 nódulos), la mayoría completos y algunos con lascados aislados, que podrían provenir del arroyo Chalía, distante $1 \mathrm{~km}$ aproximadamente. En las prospecciones realizadas en Loma Redonda, para constatar la disponibilidad de basalto informada en la hoja geológica (González, 1978) y sugerida por nosotros como fuente en trabajos anteriores (Castro Esnal et al., 2011; Castro Esnal, 2010; 2014), se corroboró que se trataba de un basalto de mala calidad para la talla por lo que se descarta que haya sido utilizado como materia prima de estas manufacturas.

Tabla 2. Artefactos formatizados, desechos (enteros + fracturados con talón) y núcleos, por materia prima. Tapera Pescán (TP) y Casa Tolkin (CT).

Table 2. Tools, debitage (complete + fragmented with striking platform) and cores, per raw material. Tapera Pescán (TP) and Casa Tolkin (CT).

\begin{tabular}{|c|c|c|c|c|c|c|c|c|}
\hline Sitio & Materia Prima & $\begin{array}{l}\text { Artefactos } \\
\text { formatizados }\end{array}$ & $\%$ & Desechos & $\%$ & Núcleos & $\%$ & $\begin{array}{l}\text { Total } \\
\text { general }\end{array}$ \\
\hline \multirow{5}{*}{ TP } & silícea & 15 & 57,7 & 10 & 38,5 & 1 & 3,8 & 26 \\
\hline & obsidiana & 3 & 25 & 9 & 75 & - & - & 12 \\
\hline & sedimentaria $\mathrm{n} / \mathrm{d}$ & - & - & 1 & 100 & - & - & 1 \\
\hline & vulcanita ácida & - & - & 2 & 100 & - & - & 2 \\
\hline & vulcanita básica & 7 & 7,6 & 73 & 79,4 & 12 & 13 & 92 \\
\hline \multirow{5}{*}{ CT } & silícea & 41 & 41,8 & 53 & 54,1 & 4 & 4,1 & 98 \\
\hline & obsidiana & 4 & 28,6 & 7 & 50 & 3 & 21,4 & 14 \\
\hline & vulcanita ácida & - & - & 4 & 100 & - & - & 4 \\
\hline & vulcanita básica & 16 & 24,2 & 43 & 65,2 & 7 & 10,6 & 66 \\
\hline & N/D & - & - & 3 & 100 & - & - & 3 \\
\hline
\end{tabular}


La presencia de los nódulos de vulcanitas básicas y la importancia de esta materia prima en las muestras de artefactos del sitio Tapera Pescán permiten considerar la hipótesis sobre la posibilidad del uso de una estrategia de aprovisionamiento de lugares (Kuhn, 1995). Esto lo sugerimos dado que, a pesar de ser una materia prima local, su distribución se encuentra relacionada con el arroyo Chalía, y por lo tanto el acceso desde Tapera Pescán implica recorrer una distancia aproximada de 1 km y subir una pendiente al regresar (Figura 2c); por lo que un acopio anticipado de nódulos, aunque se trate de distancias que implican dificultades mínimas o nulas para cazadores recolectores, minimizaría el costo de un aprovisionamiento reiterado por parte de los talladores. Sin embargo, no puede descartarse la posibilidad de que se hayan provisto de otra fuente más distante en el paisaje, con basaltos de calidad superior a los que se encuentran disponibles en el arroyo, que no haya sido detectada en nuestros relevamientos de campo. Además, es interesante realizar una comparación con el conjunto de Tapera Almeida, a aproximadamente $4 \mathrm{~km}$ en línea recta de Tapera Pescán (Figura 1 y Figura 2c), en donde, en su momento, llamó la atención el hallazgo de 18 instrumentos y 11 núcleos formatizados en vulcanita básica, en contraposición con la presencia de sólo un desecho entero, lo que indicaría que las actividades de talla sobre esta materia prima habrían sido realizadas fuera del sitio (Castro Esnal, 2014). El hecho de que Tapera Almeida (antigua vivienda de la familia Vera) esté próxima al vado del arroyo Chalía por el que discurre el actual camino que atraviesa la colonia de norte a sur, señala la posibilidad de pensar a este lugar como un punto de tránsito fluido y de fácil acceso que conecta distintas áreas de la colonia, esto explicaría tal vez la presencia de elementos que no fueron confeccionados en el sitio y la disponibilidad de una reserva de núcleos para tallar.

Al mismo tiempo, con respecto a las rocas silíceas se observa que en los nuevos conjuntos de Tapera Pescán y Casa Tolkin, la relación entre la cantidad de artefactos formatizados y los desechos no es coherente con un contexto de manufactura, ya que el número de instrumentos es alto en comparación con los desechos (Tabla 2). Esto mismo sucede en los conjuntos de Tapera Almeida y Laguna Quilchamal (Castro Esnal, 2010; 2014), lo que indica un ingreso a los sitios de artefactos silíceos en un estadio de manufactura avanzado, y refuerza la idea sugerida anteriormente (Castro Esnal, 2010; 2014) de la implementación de una estrategia de transporte de núcleos, formas base e instrumentos formatizados para el caso del sílice. Como ya se mencionó, el único sitio que presenta evidencia de formatización final, reparación y/o reactivación de instrumentos es Laguna Quilchamal.

Por su parte, la obsidiana se presenta en bajas proporciones, al igual que lo observado para Tapera Almeida y Laguna Quilchamal, en donde se postuló la aplicación de una estrategia de transporte de instrumentos formatizados, formas base y posiblemente núcleos y nódulos pequeños, y se determinó como fuente de procedencia a Pampa del Asador (Castro Esnal et al., 2011b). Las características macroscópicas de las obsidianas de los nuevos conjuntos analizados son similares por lo que se estima provendrían de la misma fuente, aunque no se descarta hacer nuevos análisis geoquímicos. Este dato ubica a El Chalía dentro de la esfera de circulación de materias primas desde Pampa del Asador tal como ocurre para otros sitios del sudoeste de Chubut en donde se ha registrado la presencia de este tipo de obsidiana (Castro Esnal, Pérez de Micou y Stern, 2017c).

\section{Consideraciones finales}

En suma, el estudio de las muestras de artefactos líticos recuperadas en los sitios Tapera Pescán y Casa Tolkin permite ampliar la información producida hasta el momento para la comprensión de los modos de habitar este espacio del sudoeste de Chubut en el pasado, previamente a los cambios acaecidos a partir del contacto europeo-indígena. Desde el punto de vista cronológico no se han encontrado nuevos indicadores que adviertan una ocupación más temprana que la anteriormente planteada, ya que en la Colonia El Chalía no se ha registrado aún la presencia de sitios en estratigrafía ni de nuevos materiales plausibles de ser datados; por lo que los fechados realizados sobre tiestos cerámicos hallados en los conjuntos de Laguna Quilchamal (aproximadamente 470 años AP) siguen siendo hasta el momento los únicos disponibles. Sin embargo, tomando en cuenta los datos cronológicos de otros sitios de la región, no se descarta la posibilidad de una ocupación más temprana del área de la colonia.

La presencia en ambos sitios de materiales de manufactura criollo-europea, tales como fragmentos de gres, metales y vidrio, y en Casa Tolkin también de lascas de vidrio y algunos posibles instrumentos sobre este material, corrobora no sólo una ocupación en tiempos de contacto, sino también la incorporación de estos elementos por parte de familias indígenas y la aplicación de técnicas tradicionales de talla sobre materiales foráneos. Esta misma situación fue reconocida anteriormente para otros sitios de El Chalía (Castro Esnal, 2010; 2014) y de Chubut (Pérez de Micou, Casanueva y Castro Esnal, 2011) y Santa Cruz (Nuevo Delaunay, 2007; Cirigliano, 2016; entre otros). Estos materiales de factura europea abren un interrogante acerca de su obtención. Considerando que la colonia está ubicada en un espacio interior, desconocido hasta fines del siglo XIX cuando los europeos lo reconocieron en compañía de los aborígenes, es altamente probable que esas manufacturas foráneas llegaran tardíamente a la región. Además de su uso como objetos completos (botellas, por ej.) la evidencia arqueológica demuestra cómo ese material no fue descartado sino, por el contrario, utilizado como nueva 
materia prima en la confección de artefactos tradicionales indígenas; un caso de ciclaje lateral según Schiffer (1990).

Por otro lado, se observa que la estrategia de aprovisionamiento de materias primas disponibles localmente, propuesta para el material lítico, se da también con respecto a los recursos vegetales. Diversos autores han planteado que la toponimia entre los cazadores patagónicos es un indicador firme de la existencia del recurso en el lugar nombrado. En este caso, el topónimo Chalía, referido a tubérculos comestibles, está marcando la disponibilidad de este recurso aprovechable durante el comienzo de la estación cálida, paliando la falta de insumos cárnicos, indicando un hito en su movilidad (Nacuzzi y Pérez de Micou, 1985; Pérez de Micou, 1994). La ubicación de estos sitios en un área de tránsito conocida para momentos históricos abre la posibilidad de pensar en un corredor por el que habrían circulado diversos grupos de cazadores recolectores hasta fines del siglo XIX, siendo este sector atractivo para detenerse desde el punto de vista de este recurso vegetal destacado y por la disponibilidad permanente de agua. La presencia de obsidiana alóctona es un indicador concreto de movimiento de grupos y además está documentada la adquisición directa de esta materia prima de Pampa del Asador como parte de una estrategia embedded (sensu Binford, 1979), al menos para el siglo XIX (Musters, 1871/1964). El pasaje de una vida nómade a una sedentaria a partir del establecimiento de la Colonia El Chalía debió transformar profundamente estas estrategias.

Finalmente, se considera que las colecciones no sistemáticas pueden aportar información válida y relevante en cuanto a diversos aspectos del comportamiento humano en el pasado, siempre y cuando se tomen los recaudos metodológicos correspondientes a la hora de interpretar los resultados y de realizar comparaciones. De este modo, a pesar de las particularidades expuestas para el caso de Casa Tolkin, los resultados señalan aspectos válidos de considerar, tales como la densidad mínima relativa de materiales, las materias primas representadas y, por último, la abundancia de raspadores de sílice que confirma, por un lado, una predilección en el uso de esta materia prima para la confección de este instrumento, $y$, por otro lado, indica la presencia de actividades de procesamiento de cueros en este sitio, tarea que se realiza hasta la actualidad.

Buenos Aires, 12 de diciembre de 2019

\section{Agradecimientos}

Dedicamos este trabajo y expresamos nuestro profundo respeto y reconocimiento al cacique Luis Quilchamal, a Aladino González y a Luisa Tolkin, fallecidos en los últimos años. Esta investigación fue financiada por subsidios de la UBA y del CONICET. Agradecemos a las familias Santibáñez y González por su gentil colaboración y hospitalidad, especialmente a Margarita, Clementina y Sebastián Santibáñez y a Justina González y al Sr. Gallardo. Silvia García, Luz Funes y Mariana Sacchi participaron de los trabajos de campo. Catharina Zönnchen colaboró en tareas de laboratorio, y Esteban Ali-Brouchoud participó en el análisis de los materiales. Finalmente, agradecemos a los evaluadores por sus sugerencias y comentarios.

\section{Bibliografía}

Aguerre, A., Andrieu, M. y lantanos, N. (2017). Arqueología en Río Mayo, sudoeste del Chubut. Excavación en el Alero Dásovich: resultados preliminares. Intersecciones en Antropología, 18, 55-65.

Aschero, C. A. (1975a). Secuencia arqueológica del Alero de las Manos Pintadas - Las Pulgas, Departamento de Río Senguer, Chubut. Relaciones, 9, 187-209.

Aschero, C. A. (1975b). Ensayo para una clasificación morfológica de artefactos líticos. Informe al CONICET. MS.

Aschero, C. A. (1983). Ensayo para una clasificación morfológica de artefactos líticos. Apéndice A y B. Cátedra de Ergología y Tecnología. Facultad de Filosofía y Letras. Universidad de Buenos Aires. MS.

Binford, L. R. (1979). Organization and formation processes: looking at curated technology. Journal of Anthropological Research 35(3):255-273.

Casanueva, M. L., Castro Esnal, A. y Pérez de Micou, C. (2019). Indígenas, colonos y colonos indígenas. Arqueología de tiempos históricos y su abordaje: una experiencia en el SO de Chubut, Patagonia Argentina. En Gómez Otero et al. (Eds.), Arqueología de la Patagonia: el pasado entre las arenas (pp. 93-104). Madryn, Conicet IDEAUS.

Castro, A., Funes, M. L. y Sacchi, M. (2007). Los pobladores del Chalía, su memoria y el registro arqueológico. Rutas indígenas y transmisión del conocimiento. En C. Pérez de Micou, S. Burry y M. Trivi de Mandri (Eds.), Aquí Vivieron (pp. 29-41). Buenos Aires, Argentina: AINA.

Castro Esnal, A. (2010). Rutas indígenas y arqueología en la provincia de Chubut (tesis Doctoral). Facultad de Filosofía y Letras, Universidad de Buenos Aires. Buenos Aires, Argentina.

Castro Esnal, A. (2014). Camino y Piedra. Rutas indígenas y arqueología en la provincia de Chubut. Buenos Aires, Argentina: Fundación Félix de Azara.

Castro Esnal, A., Sacchi, M. y Pérez de Micou, C. (2011a). Aspectos generales de la tecnología lítica de los sitios de Colonia El Chalía (SO de la provincia de Chubut, 
A. Castro Esnal et al.| Revista del Museo de Antropología 13 (3): 27-38 | 2020

DOI: http://doi.org/10.31048/1852.4826.v13.n3.27025

Argentina). International Journal of Southamerican Archaeology, 9, 28-40.

Castro Esnal, A., Pérez de Micou, C. y Stern, C. (2011b). Circulación de obsidiana en Chubut, Patagonia Central, Argentina: uso de las materias primas extra-regionales como indicadores de movilidad e interacción entre grupos cazadores recolectores. Revista do Museo e Arqueología y Etnologia, 21, 93-102.

Castro Esnal, A., Pérez de Micou, C. y Casanueva, M. L. (2017a). Early Holocene occupation of the forest-steppe ecotone of southern South America: evidence from Casa de Piedra de Roselló cave (Chubut, Patagonia Argentina). PaleoAmerica, 3, 276-282.

Castro Esnal, A., Stern, C. y Pérez de Micou, C. (2017b). Aplicación de estudios geoquímicos sobre artefactos de obsidiana procedentes de contextos estratigráficos y superficiales en Aldea Beleiro, SO de Chubut (Patagonia, Argentina). Magallania, 45(1), 141-153.

Castro Esnal, A., Pérez de Micou, C. y Stern, C. (2017c, 7-12 de noviembre). Obsidian Provenance and Transport in Central Patagonia (Chubut, Argentina): From the Early Holocene to Historical Contexts [Poster]. $11^{\text {th }}$ International Symposium on Knappable Materials "From toolstone to stone tools", Buenos Aires, Argentina.

Cirigliano, N. A. (2016). Movilidad de grupos indígenas y aprovechamiento de materias primas entre el extremo sur del macizo del Deseado y la cuenca del Río Santa Cruz durante los últimos 2000 años (Provincia de Santa Cruz, Argentina) (tesis Doctoral). Facultad de Filosofía y Letras, Universidad de Buenos Aires, Buenos Aires, Argentina.

Dal Molin, C.N., (1998). Alto Río Senguer. Programa Nacional de Cartas Geológicas de la República Argentina. Hoja Geológica 4572-IV. Boletín 255. Buenos Aires, Argentina: Servicio Geológico Minero Argentino. Instituto de Geología y Recursos Minerales.

De la Vaulx, H. [1901] (2008). Viaje a la Patagonia 1896. Traducido por F. Coronato. Puerto Madryn, Argentina: Asociación Punta Cuevas, Fundación Ameghino.

Escalada, F. A. (1949). El complejo tehuelche. Buenos Aires, Argentina: Coni.

Espinosa, S. L. y Goñi, R. A. (1999). ¡Viven!: una fuente de obsidiana en la provincia de Santa Cruz. En Soplando en el viento... Actas de las Terceras Jornadas de Arqueología de la Patagonia (pp. 177-188). Buenos Aires-Neuquén, Argentina: INAPL-Universidad Nacional del Comahue.

Fernández Garay, A. y Hernández, G. (1999). Origen y uso del fuego. Mito recogido entre los tehuelches araucanizados de la Patagonia argentina. Amerindia,
24, 73-90.

Fontana, L. J. (1999 (1886)). Viaje de Exploración en la Patagonia Austral. Buenos Aires, Argentina: Confluencia.

García, S. P. (2009). Educación institucional y educación familiar. El caso de la Reserva del Chalía en la provincia de Chubut. En C. Pérez de Micou, M. Trivi de Mandri y L. S. Burry (Eds.), Imágenes desde un alero. Investigaciones multidisciplinarias en Río Mayo, Chubut. Patagonia Argentina (pp. 163-174). Buenos Aires, Argentina: Fundación Félix de Azara.

García, S. P. y Moreno, E. (2010). De los toldos a la casa de material. La oralidad como instrumento para interpretar las transformaciones en la vivienda de grupos originarios de la Patagonia. En América Malbrán Porto y Enrique Méndez Torres (Coords.), Memorias del I Congreso de Folklore y Tradición Oral en Arqueología (pp. 157176). Ciudad de México, México: Escuela Nacional de Antropología e Historia.

Gradin, C. J. y. Aschero, C. A. (1978). Cuatro fechas radiocarbónicas para el Alero del Cañadón de las Manos Pintadas. Relaciones, 12, 245-248.

González, R.L. (1978). Descripción Geológica de las Hojas 49a, Lago Blanco y 49b, Paso Río Mayo. Boletín 154155, 1-45. Buenos Aires, Argentina: Servicio Geológico Nacional.

Kuhn, S. (1995). Mousterian Lithic Technology. An Ecological Perspective. Princeton, Estados Unidos: Princeton University Press.

Mena, F., Lucero, V., Reyes, O., Trejo, V. y Velásquez, H. (2000). Cazadores Tempranos y Tardíos en la Cueva Baño Nuevo-1, margen occidental de la estepa centropatagónica (XI Región de Aisen, Chile). Anales Instituto Patagonia, Serie Ciencias Humanas, 28, 173-195.

Mena, F., Reyes, O., Stafford, T. y Southon, J. (2003). Early Human Remains from Baño Nuevo-1 (Central Patagonian Andes; Chile). Quaternary International,109-110, 113-121.

Méndez, C., Reyes, O., Nuevo Delaunay, A., Trejo, V., Barberena, R. y Velásquez, H. (2011). Ocupaciones humanas en la margen occidental de Patagonia Central: Eventos de poblamiento en alto río Cisnes. Magallania, 39 (2), 223-242.

Méndez, C., Nuevo Delaunay, A., Reyes, O., Ozán, I. L., Belmar, C. y López, P. (2018). The initial peopling of Central Western Patagonia (southernmost South America): Late Pleistocene through Holocene site context and archaeological assemblages from Cueva de la Vieja site. Quaternary International, 473, 261-277. 
Mengoni Goñalons, G., Figuerero Torres, M. J., Fernández, M. V. y Chávez, P. V. (2009). Carácter de las ocupaciones humanas en el área de Los Antiguos- Monte Zeballos y Paso Roballos (Santa Cruz, Argentina). En M. Salemme et al. (Eds.), Arqueología de la Patagonia - Una Mirada desde el último confín (pp. 1061-1074). Ushuaia, Argentina: Editorial Utopías.

Mengoni Goñalons, G., Fernández, M. V. y Figuerero Torres, M. J. (2013). Tiempo y movilidad en el área de Los Antiguos-Monte Zeballos y Paso Roballos, Santa Cruz, Argentina. En A. Zangrando et al. (Eds.), Tendencias teórico-metodológicas y casos de estudio en la arqueología de la Patagonia (pp. 441-449). San Rafael, Argentina: Museo de Historia Natural de San Rafael.

Miceli, J. y Guerrero, S. (2007). Redes libres de escala y su uso en el análisis de datos etnográficos: El caso de la comunidad Tehuelche del Chalía. En Grupo Antropocaos, Exploraciones en antropología y complejidad (pp. 177191). Buenos Aires, Argentina: Editorial SB.

Moreno E., Pérez, H. y Ramírez, F. (2016) Esquema cronológico y evolución del paisaje en el Bajo de Sarmiento (Chubut). En F. Mena (Ed.), Arqueología de la Patagonia: de mar a mar (pp. 477 - 485). Coyhaique, Chile: Nire Negro

Muñiz, M. y Perea, E. (2000). La Reserva del Chalía, la tierra del cacique Kéltchamn y su tribu. Río Mayo, Chubut, Argentina. MS.

Musters, G. 1964 (1871). Vida entre los patagones. SolarHachette. Bs. As.

Nacuzzi, L. y Pérez de Micou, C. (1985). Los recursos vegetales de los cazadores de la Cuenca del Río Chubut. Cuadernos del INAPL, 10, 407-424.

Nuevo Delaunay, A. (2007). Tecnología vítrea en el siglo XX, Lago Strobel (Santa Cruz, Argentina). En F. Morello, M. Martinic, A. Prieto y G. Bahamonde (Eds.), Arqueología de Fuego-Patagonia. Levantando piedras, desenterrando huesos...y develando arcanos (pp. 853-859). Punta Arenas, Chile: CEQUA.

Onelli, C. (1998). Trepando los Andes. Buenos Aires, Argentina: El Elefante Blanco.

Pérez de Micou, C. (1994). La Etnohistoria en los estudios Paleoetnobotánicos de cazadores recolectores. Presentación de un caso. Cuadernos del INAPL, 15, 225-236.

Pérez de Micou, C., Sacchi, M., Castro, A. y Funes, M.L. (2009). Estudios de Arqueología en la Colonia Indígena de Chalía, Dpto. Senguer, Chubut. En P. F. Azar, E. M. Cúneo y S. N. Rodríguez (Eds.), Tras la senda de los ancestros:
Arqueología de Patagonia (pp.117-127). San Carlos de Bariloche. Argentina: Universidad Nacional del Comahue.

Pérez de Micou, C., Casanueva, M. L. y Castro, A. (2011). "Campo Oses" (provincia de Chubut). Indígenas y colonos europeos, distintas formas de habitar un mismo espacio. Intersecciones en Antropología, 12, 333-343.

Pinotti, L. (2001). Sin embargo existimos. Reproducción biológica y cultural de una comunidad tehuelche. Buenos Aires, Argentina: EUDEBA.

Pinotti, L. (2004). Aquellos Tehuelches. Buenos Aires, Argentina: Proyecto Editorial.

Reyes, O., Méndez, C., Trejo, V. y Velásquez, H. (2007). El Chueco 1: Un asentamiento multicomponente en la estepa occidental de Patagonia Central (11400 a 2700 años cal AP, 44 S). Magallania, 35(1), 61-74.

Rizzo, F., Scheinsohn, V. y Leonardt, S. (2016). Registro arqueológico a cielo abierto en las cuencas de los ríos Genoa y Pico. En F. Mena (Ed.), Arqueología de la Patagonia: de mar a mar (pp. 277-286). Coyhaique, Chile: Ñire Negro.

Roberts, T. y Gavirati, M. (Comps.) (2008). Diarios del explorador Llwyd ap Iwan. El desvío del río Fénix y la colonia galesa de Santa Cruz que pudo ser. Villa Adelina, Buenos Aires, Argentina: Patagonia Sur Libros-La Bitácora Editores.

Sacchi, M. (2012). Materias Primas Líticas y Redes Sociales entre los grupos cazadores-recolectores de Patagonia Centro-Meridional (tesis Doctoral). Facultad de Filosofía y Letras, Universidad de Buenos Aires. Buenos Aires, Argentina.

Sacchi, M., Funes, M. L., y Castro, A. (2009). Testimonios orales y Arqueología, una primera aproximación. En: M. Salemme, F. Santiago, M. Alvarez, E. Piana, M. Vázquez y E. Mansur (Comps.), Arqueología de Patagonia: una mirada desde el último confín (Tomo I, pp. 207-213). Ushuaia, Argentina: Editorial Utopías.

Scheinsohn, V., Rizzo, F. y Leonardt, S. (2010). Investigaciones arqueológicas en el área Centro-Oeste de Chubut. En J. R. Bárcena y H. Chiavazza (Eds.), Arqueología Argentina en el bicentenario de la revolución de Mayo (tomo V, pp. 1981-1985). Mendoza, Argentina: Universidad Nacional de Cuyo.

Scheinsohn, V., Dahinten, S. L., Gómez Otero, J., Rizzo, F., Leonardt, S., Tchilinguirián, P., Beroqui, B. (2017). La antigüedad de la ocupación humana en el centrooeste del Chubut: nuevos datos del valle del Genoa. Arqueología, 23(1), 109-124. 
Schiffer, M. B. (1990). Contexto arqueológico y contexto sistémico. Boletín de Antropología Americana 22:81-93.

Soriano, A. (1956). Los distritos florísticos de la Provincia Patagónica. Revista de Investigaciones Agrícolas, 10 (4), 323-347.

Stern, C. R. (1999). Black Obsidian from central-south Patagonia, chemical characteristics, sources and regional distribution of artifacts. En Soplando en el viento. Actas de las III Jornadas de Arqueología de la Patagonia (pp. 221-234). Buenos Aires-Neuquén, Argentina: INAPLUniversidad Nacional del Comahue.
Stern, C. (2004). Obsidian in Southern Patagonia: review of the current information. En M. T. Civalero, P. Fernández y G. Guráieb (Comps.), Contra Viento y Marea. Arqueología de Patagonia (pp.167-178). Buenos Aires, Argentina: INAPL, SAA.

Stern, C., Castro Esnal, A., Pérez de Micou, C., Méndez, C. y Mena, F. (2013). Circulación de Obsidianas en Patagonia Central-Sur entre 44 y $46^{\circ} \mathrm{S}$ (Chubut, Argentina, y Coyhaique y Alto Cisnes, Chile). En Zangrando et al. (Comps.), Tendencias teórico-metodológicas y casos de estudio en la Arqueología de la Patagonia (pp. 243-250). San Rafael, Argentina: Museo de Historia Natural de San Rafael. 